\title{
Progressive Transmission of Medical Images via a Bank of Generative Adversarial Networks
}

\author{
Ching-Chun Chang $\mathbb{D}^{1},{ }^{1}$ Xu Wang $(\mathbb{D})^{2}$ Ji-Hwei Horng $\mathbb{D}^{3},{ }^{3}$ and Isao Echizen $\mathbb{D}^{1}$ \\ ${ }^{1}$ National Institute of Informatics, Tokyo, Japan \\ ${ }^{2}$ Department of Information Engineering and Computer Science, Feng Chia University, Taichung 40724, Taiwan \\ ${ }^{3}$ Department of Electronic Engineering, National Quemoy University, Kinmen 89250, Taiwan
}

Correspondence should be addressed to Xu Wang; xu.wang.phd@gmail.com

Received 13 March 2021; Revised 29 March 2021; Accepted 9 April 2021; Published 28 April 2021

Academic Editor: Chi-Hua Chen

Copyright ( 92021 Ching-Chun Chang et al. This is an open access article distributed under the Creative Commons Attribution License, which permits unrestricted use, distribution, and reproduction in any medium, provided the original work is properly cited.

\begin{abstract}
The healthcare sector is currently undergoing a major transformation due to the recent advances in deep learning and artificial intelligence. Despite a significant breakthrough in medical imaging and diagnosis, there are still many open issues and undeveloped applications in the healthcare domain. In particular, transmission of a large volume of medical images proves to be a challenging and time-consuming problem, and yet no prior studies have investigated the use of deep neural networks towards this task. The purpose of this paper is to introduce and develop a deep-learning approach for the efficient transmission of medical images, with a particular interest in the progressive coding of bit-planes. We establish a connection between bit-plane synthesis and image-to-image translation and propose a two-step pipeline for progressive image transmission. First, a bank of generative adversarial networks is trained for predicting bit-planes in a top-down manner, and then prediction residuals are encoded with a tailored adaptive lossless compression algorithm. Experimental results validate the effectiveness of the network bank for generating an accurate low-order bit-plane from high-order bit-planes and demonstrate an advantage of the tailored compression algorithm over conventional arithmetic coding for this special type of prediction residuals in terms of compression ratio.
\end{abstract}

\section{Introduction}

With the development of digital imaging equipment, more and more medical images have been produced and a medical image dataset usually contains a large number of images. Transmission of abundant medical images over a low narrow bandwidth public network will no doubt cause critical network pressure and further delay the timing of diagnosis [1].

To increase the speed of medical image transmission, some schemes have been proposed to build up more effective special networks. For instance, in [2], a unique pair of interfaces was proposed to provide a fast thoroughfare for medical data transmission between two DICOM applications and could speed up 1.22 and 13 times, respectively, in the local area networks (LAN) and wide area networks (WAN). Later, they further proposed an improved network [3] to combine the local area network (LAN) and wide area network (WAN) and used parallel TCP connections to optimize the DICOM protocol. This network can speed up about 2.2 to 3.5 times when transmitting magnetic resonance images.

On the other aspect, some new image transmission techniques have been proposed. An intuitive way is to reduce the resolution of medical images before transmission in a poor bandwidth network. However, doctors suffer from misdiagnosis with the lower resolution medical images. Therefore, the progressive image transmission (PIT) [4] technique emerges as the times require. The conventional raster scan ordered pixel by pixel image transmission 
technique is inefficient, and the key region of the image which indicates the disease may be received at the end of the transmission makes doctors out of patience and affects the treatment. The progressive image transmission technique usually transmits a lower resolution image at first and gradually transmits the details of the image to further increase the resolution until the original image is totally transmitted. Through the transmission, receivers can decide whether they want to receive the details of the image or not in a short time. As a consequence, doctors can control the final image resolution and the required storage space. Over the past decades, some mature PIT techniques have been proposed. The bit-plane progressive image transmission method (BPM) $[5,6]$ is one of the low computational complexity and intuitive technique to transmit the image bit-plane by bit-plane which is detailed in Section 2. Another sort of progressive image transmission techniques is that, in [7-12], the image is divided into nonoverlapping blocks, and a quantized pixel value is used to represent the whole block, then, progressively dividing the image into finer blocks to improve the image resolution. Some schemes [13-17] transform the image into a wavelet domain and transmit the coefficients instead of pixels. Lossy compression [18] and lossless compression [19] methods are also adopted in some schemes to reduce the file size in each round of transmission.

The deep learning technique has matured in recent years and drawn massive research which is widely used in industry [20]. Some advanced deep learning architectures have also been introduced into medical image processing. Medical image classification is the most important area where deep learning makes a great contribution. The CNN network has been widely used in medical image classification and achieved a higher accuracy [21-23]. The deep learning technique is also suitable for medical image detection. The methods for organ, region, and landmark localization [24-26] and object and lesion detection [27-29] all adopt the deep learning architectures. Then, the medical image segmentation is naturally developed in the detected images [30-32]. The other tasks on medical images processing such as content-based image retrieval [33]; image generation and enhancement [34]; and description of medical images with shape, margin, and density [35] have also been proposed. Recently, the deep learning architecture with adversarial learning: generative adversarial network (GAN) has been proposed by Goodfellow et al. [36] that has the ability to generate realistic-looking images. Based on the GAN network, a powerful architecture pix2pix [37] was set up as an image bit-plane predictor to translate the image styles and has been adopted in different scenarios such as steganography [38] and even in medical images [39, 40]. However, so far, these deep learning techniques have not been applied to the medical image transmission. By leveraging the prediction ability of the GAN network, the medical images can be compressed before transmission and thus the efficiency of transmission can be improved.

In this paper, we adopt the framework of the bit-plane PIT method to transmit medical images. To reduce the amount of data before transmission, a GAN-based predictor bank is designed to predict the bit-planes. By progressively predicting the bit-plane to be sent based on previously transmitted bit-planes, we need only to send the residual provided that the receiver can execute the same prediction. The proposed GAN-based predictor bank produces a prediction of high accuracy so that the residual can be efficiently compressed before transmission. Thus, the efficiency of bitplane PIT is greatly improved, and the transmission cost can be reduced. The main contributions of our scheme are summarized as follows:

(1) Introduce a deep neural network of pix2pix GAN network into the research of progressive medical image prediction and transmission

(2) Design a GAN-based predictor bank to help bitplane compression of medical images

(3) Propose an adaptive residual bit-plane compression technique of high compression ratio

Experimental results validate the effectiveness and efficiency of the proposed prediction network and compression technique. The rest of this paper is organized as follows. Section 2 briefly introduces the bit-plane method of the progressive image transmission. Section 3 details the proposed adversarial learning for progressive bit-plane prediction scheme and the adaptive residual bit-plane compression technique. Section 4 provides the experimental results to demonstrate the accuracy of our prediction scheme and the efficiency of the proposed compression technique. In addition, the performance of the proposed residual bit-plane compression is compared with the conventional arithmetic coding. The conclusions are offered in Section 5.

\section{Bit-Plane Method of PIT}

In this section, the bit-plane method of progressive image transmission (BPM) technique which has been widely used to transmit a large volume of high-resolution medical images, is briefly introduced. The progressive image transmission (PIT) technique is an effective way to transmit images over narrow bandwidth channels. Among the PIT techniques, BPM is a simple and intuitive way of transmission, which divides an image into bit-planes and sends it plane by plane. For the grayscale images, each pixel is valued from 0 to 255, that is, 256 gray levels, and can be represented by 8 bits. The original image can be decomposed into eight bit-planes from the most significant bit-plane $b_{8}$ to the least significant bit-plane $b_{1}$, so that $b_{8}$ records the main content and $b_{1}$ records the subtle details, respectively, of the original image. Therefore, in the bit-plane PIT method, bit-planes from $b_{8}$ to $b_{1}$ are sequentially transmitted and the receiver can progressively recover the image until all bit-planes are sent or the current image quality is satisfied. The detailed BPM method is described below.

In the most significant bit-plane $b_{8}$, the bit "0" and " 1 " indicate the corresponding pixels are ranging from " 0 to 127 " and "128 to 255 ," respectively. Therefore, after receiving $b_{8}$, the mean values of these two intervals, that is, 64 and 192, are used to coarsely represent them. When receiving the next bit-plane $b_{7}$, the receiver updates the image by using finer 
quantization levels of $(32,96,160,224)$ to represent corresponding codes “ 00 ," “ 01 ," “ 10 ," and " 11 ." This process proceeds until all of the eight bit-planes are sent. An example of BPM transmission is illustrated in Figure 1, where the progressive combination of bit-planes and their corresponding recovered images are presented. As the number of received bit-planes increases, the available quantization levels also increase and a finer recovered image can be obtained.

\section{Progressive Bit-Plane Prediction and Compression}

Based on BPM progressive image transmission, we propose a bit-plane prediction method and a compression technique to improve the transmission efficiency of medical images. In this section, the proposed image transmission scheme and a pix2pix model-based bit-plane prediction method are introduced first. Then, a new technique for compression of the prediction error bit-plane is presented.

\subsection{Adversarial Learning for Progressive Bit-Plane Prediction.} For the purpose of progressively predicting image bit-plane by bit-plane, the generative adversarial network is introduced in this paper to predict and synthesize bit-planes. GAN architecture is a classical neural network to generate synthetic images of a specific style through training. The pix2pix model is a famous model of a GAN-based network which can be exploited to make an image-to-image translation. In the pix2pix model, the generator $G$ is a U-Net, which is designed for biomedical image segmentation, instead of the conventional encoder-decoder net. The U-Net consists of a contracting path to downsample the input image, and an expansive path to generate an output image of full resolution. The contracting path consists of four steps connected by $2 \times 2$ max pooling layer with stride 2 ; each step contains three repeated $3 \times 3$ convolutions with ReLU. The expansive path also consists of four steps connected by $2 \times 2$ upconvolution and halves of the feature maps are cropped from the contracting path. For the discriminator, the PatchGAN is adopted to discriminate that the overlapping patches in the generated image are real or fake. Different from the conventional GAN discriminator which only output one result, that is, real or fake, PatchGAN outputs a matrix and each element reflects the pixel differences of the current patch between the synthetic image and the original image in the current patch region. Additionally, to obtain a generated image with higher similarity to the input original image, the Manhattan distance $\left(\ell_{1}\right.$ norm $)$ which is more suitable for high dimensional images is chosen to evaluate the difference between input and output images. Therefore, in the pix2pix model, assume $x$ and $y$ are the input and output images; the difference between input and output is evaluated by

$$
\mathscr{L}_{L 1}(G)=\mathbb{E}\left[\|y-G(x)\|_{1}\right] .
$$

Then, the final objective is obtained by mixing the $\ell_{1}$ loss with the traditional GAN loss by

$$
\begin{aligned}
G^{*} & =\arg \min _{G} \max _{D} \mathscr{L}_{\mathrm{GAN}}(G, D)+\lambda \mathscr{L}_{L 1}(G), \\
\mathscr{L}_{\mathrm{GAN}}(G, D) & =\mathbb{E}[\log D(y)]+\mathbb{E}[\log (1-D(G(x)))],
\end{aligned}
$$

where $\lambda$ is a hyperparameter to balance two losses to achieve better performances.

Through the above architecture, the pix 2 pix model can translate the input image into different styles such as color images, day-to-night, and image inpainting. Inspired by pix2pix image-to-image translation model, in this paper, we train the pix 2 pix model to predict bit-planes of medical images. The proposed scheme is based on the BPM of progressive image transmission. A medical image is divided into eight bit-planes and transmitted plane by plane. On the receiver side, bit-planes are also received plane by plane and the medical image can be progressively reconstructed. During transmission, we leverage the pix 2 pix model to generate a predicted version of the bit-plane to be sent based on the previous bit-planes. Benefiting from the high accuracy of prediction, the prediction error of bit-plane (residual bit-plane) can be compressed efficiently. Thus, a bit-plane can be transmitted by sending its residual instead. The predictors of different layers of bit-plane are trained separately, organized into a predictor bank, and shared with the receiver. When receiving the residual, the receiver can do the same prediction to obtain the predicted bit-plane, and then, the original bit-plane can be recovered according to the received residual bit-plane and the predicted bit-plane. The framework of our proposed progressive medical image transmission scheme is shown in Figure 2.

In detail, assuming the bit-planes from the MSB to LSB are $b_{8}$ to $b_{1}$, we feed all $b_{8}$ bit-planes of training images into the model as the input images to generate output images and set their corresponding $b_{7}$ bit-planes as the ground truth images. The standard format of input and output for the pix2pix model is an image of 8-bit depth. To fit this format, zeros are appended behind the provided bit-planes. When training the model for predicting $b_{6}, b_{8}$ and $b_{7}$ are concatenated as the input. An illustration for this case is shown in Figure 3. The detailed prediction models for the remaining bit-planes are trained in a similar manner. Then, a series of pretrained predictors are organized into a predictor bank as shown in Figure 4, which contains seven predictors for predicting bit-planes of different layers. For instance, the $b_{6}$ predictor can predict bit-plane $b_{6}$ by using the bit-planes $b_{8}$ and $b_{7}$. Therefore, each bit-plane from $b_{7}$ to $b_{1}$ can be progressively predicted by its previous bit-planes. On the application phase, the residual bit-plane can be calculated by comparing the bit-plane to be sent and the synthetic one by

$$
\mathscr{R}_{i, j}= \begin{cases}0, & \text { if } \mathscr{B}_{i, j}=\mathcal{S}_{i, j}, \\ 1, & \text { if } \mathscr{B}_{i, j} \neq \mathcal{S}_{i, j},\end{cases}
$$

where $\mathscr{R}, \mathscr{B}$, and $\mathcal{S}$ represent the residual, original, and synthetic bit-planes, respectively. Assuming the image is sized $M \times M, i, j$ denote the coordinates and range from 1 to $M$. In this paper, the seven residual bit-planes are denoted by symbols $r_{7}$ to $r_{1}$. On the receiver side, the predicted bit-plane 


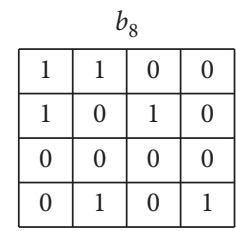

\begin{tabular}{|l|l|l|l|}
\hline 192 & 192 & 64 & 64 \\
\hline 192 & 64 & 192 & 64 \\
\hline 64 & 64 & 64 & 64 \\
\hline 64 & 192 & 64 & 192 \\
\hline \multicolumn{4}{|c|}{$(64,192)$}
\end{tabular}

Original image

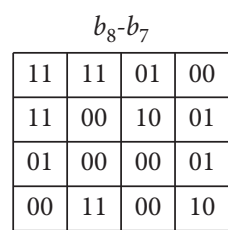

\begin{tabular}{|l|l|l|l|}
\multicolumn{5}{c}{$b_{8}-b_{6}$} \\
\hline 110 & 111 & 011 & 000 \\
\hline 110 & 001 & 100 & 011 \\
\hline 010 & 001 & 001 & 010 \\
\hline 001 & 110 & 000 & 101 \\
\hline
\end{tabular}

… $\quad b_{8}-b_{1}$

\begin{tabular}{|c|c|c|c|}
\hline 224 & 224 & 96 & 32 \\
\hline 224 & 32 & 160 & 96 \\
\hline 96 & 32 & 32 & 96 \\
\hline 32 & 224 & 32 & 160 \\
\hline
\end{tabular}

$(32,96,160,224)$

\begin{tabular}{|c|c|c|c|}
\hline 208 & 240 & 112 & 16 \\
\hline 208 & 48 & 144 & 112 \\
\hline 80 & 48 & 48 & 80 \\
\hline 48 & 208 & 16 & 176 \\
\hline
\end{tabular}

$(16,48,80,112$,

$144,176,208,240)$

Recovered images

Figure 1: An illustration of the bit-plane PIT method.

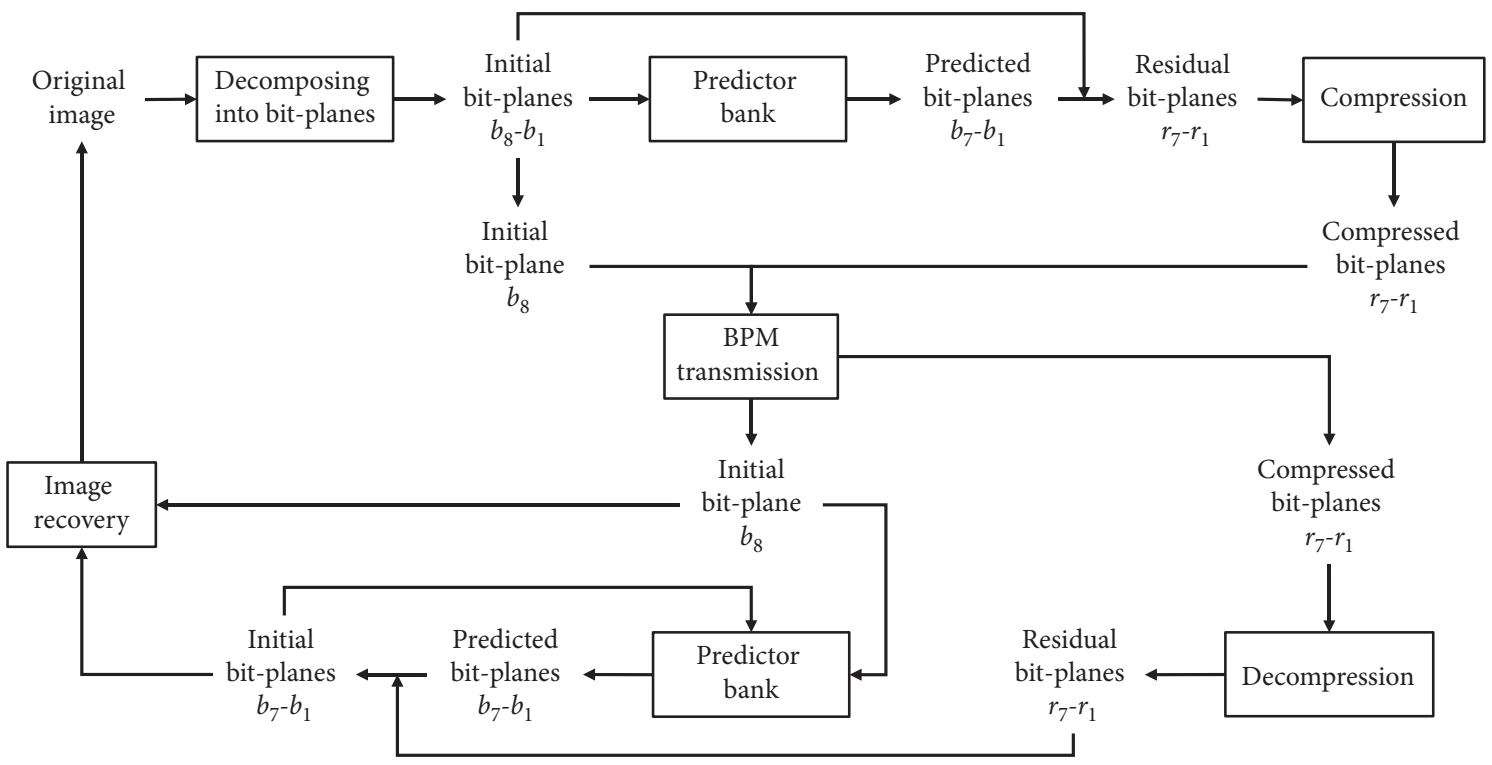

FIgURE 2: The framework of our proposed scheme.

can be obtained by the preshared predictor bank. After receiving the residual bit-plane, the real bit-plane can be recovered by

$$
\mathscr{B}_{i, j}= \begin{cases}\mathcal{S}_{i, j}, & \text { if } \mathscr{R}_{i, j}=0, \\ \overline{\mathcal{S}_{i, j}}, & \text { if } \mathscr{R}_{i, j}=1,\end{cases}
$$

where $\overline{\delta_{i, j}}$ denotes the binary bit-flipped value of $\delta_{i, j}$, that is, change "1" to " 0 " or change " 0 " to "1."

3.2. Adaptive Residual Bit-Plane Compression. The size of the prediction residual and its original bit-plane is the same. The major difference is that the values in the residual bit-plane are mostly zero. So, it can be compressed more efficiently. In addition, the error bits of our GAN-based bit-plane prediction are highly concentrated in the vicinity of image edges or complex details. According to the features of the residual bit-planes, we further propose an adaptive compression technique to reduce the data size. The detailed procedures are as follows:

Step 1: decompose a residual bit-plane $\mathscr{R}$ sized $M \times M$ into $M^{2} / m^{2}$ nonoverlapping blocks $\mathscr{R}^{n}$ sized $m \times m$, where $n$ ranges from 1 to $M^{2} / m^{2}$.

Step 2: initialize a location map $L$ with the length of $M^{2} / m^{2}$.

Step 3: scan the residual blocks in the raster scan order. If values in the block $\mathscr{R}^{n}$ are all "0" or all " 1 ," go to Step 4; otherwise, go to Step 5.

Step 4: set the $n$-th bit $L^{n}$ of the location map $L$ to "0," and record the block value by one bit, that is, $z^{n}=\{b\}$, 


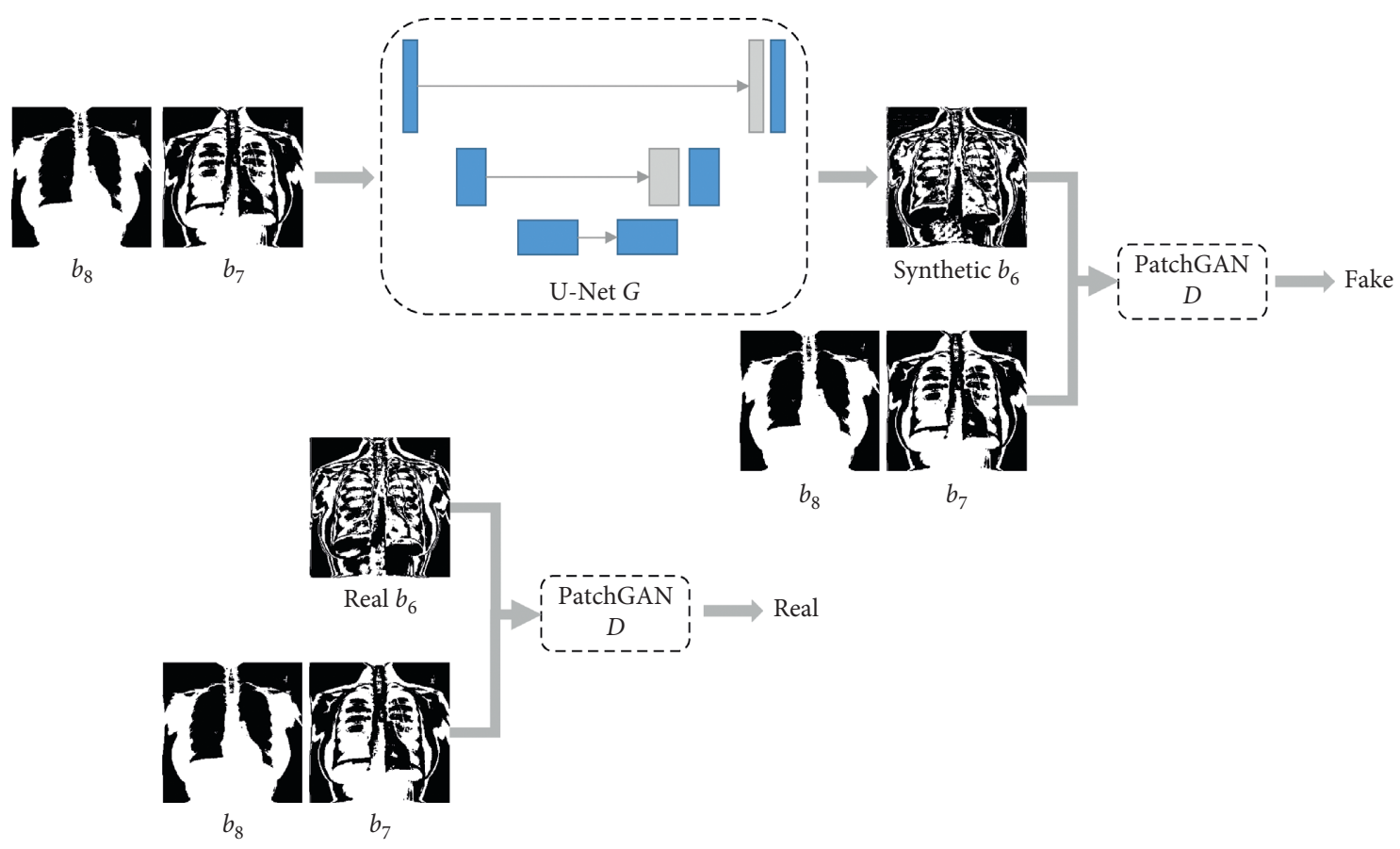

Figure 3: The training workflow of the network architecture.

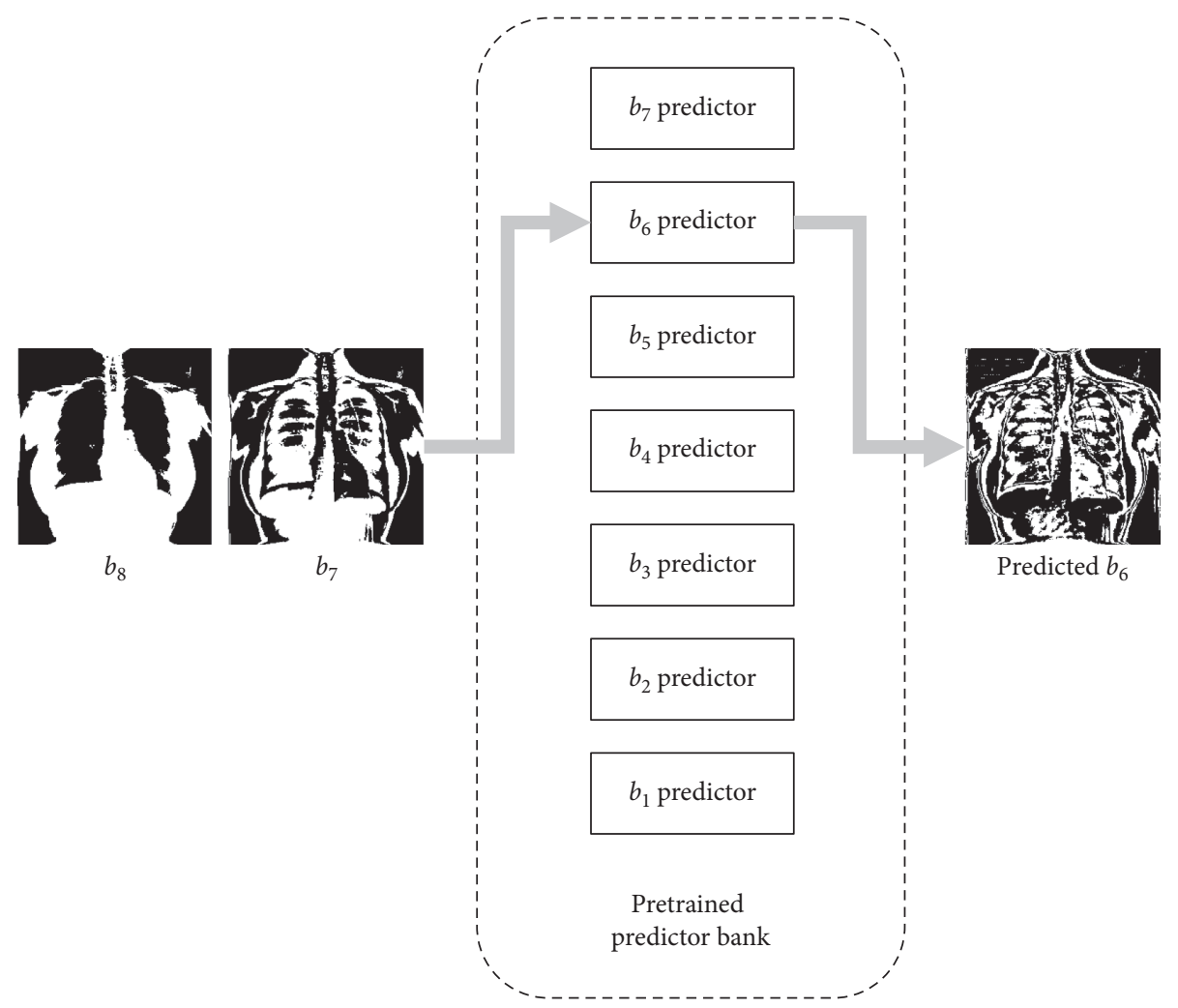

FIgURE 4: The predictor bank for bit-plane prediction.

where $z^{n}$ denotes the $n$-th segment of the recovery sequence and $b$ is the common value of the block's elements.
Step 5: set $L^{n}$ to " 1 ," and record all $m^{2}$ bits of the current residual block into the recovery sequence $r^{n}$ in raster scan order. 
Step 6: return to Step 3 until all blocks have been scanned to obtain the location map $L$ and the recovery sequence $r$.

Step 7: compress the location map $L$ by arithmetic coding to acquire the final location map $L^{\prime}$. Then, the final compressed residual bit-plane is obtained by concatenating $L^{\prime}$ and $r$.

When receiving the compressed residual bit-plane, the initial residual bit-plane can be obtained by the following decompression procedures:

Step 1: decompress the location map $L^{\prime}$ by arithmetic decoding to obtain the $M^{2} / m^{2}$ sized location map $L$.

Step 2: if $L^{n}$ is "0," all bits in the current block $\mathscr{R}^{n}$ are the same and go to Step 3; otherwise, go to Step 4.

Step 3: extract one-bit $z^{n}$ from the recovery sequence $r$ and set all $m^{2}$ bits in $\mathscr{R}^{n}$ to $r^{n}$.

Step 4: extract $m^{2}$ bits $z^{n}$ from $r$ and rearrange these bits in the raster scan order to recover $\mathscr{R}^{n}$.

Step 5: return to Step 2 until all blocks have been scanned. Then, the initial residual bit-plane $\mathscr{R}$ can be obtained by tiling the recovered residual blocks in the raster scan order.

A simple example is given in Figure 5. In this example, the block size $m$ is set to 2 . Since the first, third, and fourth blocks are uniform valued blocks, the corresponding bits in the location map are set to 0 . Meanwhile, just one sample bit is recorded into the recovery sequence for each of them. For the rest blocks, their corresponding bits in the location map are set to 1 and all bit values are recorded. When recovering these blocks, referring to the location map and the recovery sequence, all blocks can be totally recovered.

\section{Experimental Results}

In this section, we evaluate the performance of our proposed bit-plane prediction scheme with generative adversarial networks and compare our proposed residual bit-plane compression scheme with the conventional arithmetic coding compression technique. The dataset used for training and testing, the training details, and the evaluation metrics are first introduced, and then, the performance of the proposed scheme is evaluated.

4.1. Dataset and Training Details. The image samples for training and testing are the greyscale chest X-ray images collected from the National Institutes of Health under the U.S. Department of Health and Human Services. In our experiments, 10,000 images from the first compressed file are used for training, and the other 10 images from the fifth compressed file as shown in Figure 6 are used for performance evaluating and analyzing. Before training and testing, all the images are first downsampled into $256 \times 256$ sized images to fit our proposed network architecture.

When training the proposed model, each bit-plane prediction is trained over 100 epochs and the initial learning rate and the batch size are set to $2 \times 10^{-4}$ and 32 , respectively. The learning rate is enforced halfway during training and the hyperparameter $\lambda$ is set to $10^{3}$. The model parameters are updated and optimized by the Adam function.

4.2. Evaluation Metrics. To evaluate the performance of the predicted bit-planes, two important metrics, error rate (ER) and the compression ratio (CR), are introduced in this paper to conduct some experiments. For a predicted bit-plane, referring to its original bit-plane, if the two bits in the same place are the same, the corresponding bit in the residual bitplane is set to " 0 " and set to " 1 " when the predicted bit is incorrect. The error rate (ER) is defined as the ratio of " 1 's" in the residual bit-plane and can be calculated by

$$
\mathrm{ER}=\frac{\varepsilon}{M \times M}(\%)
$$

where $\varepsilon$ denotes the number of error bits. After obtaining the residual bit-plane, different compression techniques are adopted to compress it. The compression ratio (CR) denotes the ratio between the data sizes of the original and the compressed versions that can be calculated by

$$
\mathrm{CR}=\frac{M \times M}{\delta},
$$

where $\delta$ represents the data size, in bits, of the compressed residual. Generally, the compression ratio is greatly dependent on the error rate, and a residual of a low error rate can be compressed more efficiently. Thus, a prediction of high accuracy can improve the transmission efficiency.

4.3. Performances. First, the test image "Image 05 " is used to demonstrate the experimental results of the original bitplanes, prediction bit-planes, and corresponding residual bit-planes.

From Figure 7, when receiving the bit-plane 8 , the rest bit-planes can be progressively synthesized to obtain the predicted bit-planes. In the residual bit-plane, the white pixels are the error predictions, and most of them are concentrated in the edges of the image. The error rate of the bit-plane 7 is the lowest because this bit-plane is relatively smooth and just a few edges are presented. The error rate gradually increases as the details of a bit-plane become more complex. Nevertheless, the error rate of bit-plane 1 is still lower than $35 \%$ and most bits can be accurately predicted. Therefore, the proposed scheme can progressively predict bit-planes with high accuracy. To further verify the prediction accuracy, Table 1 lists all error rates of different layers in ten test images.

The error rate monotonously increases from $r_{7}$ to $r_{1}$. Two exceptions occur in "Image 04" and "Image 06": the error rate of $r_{6}$ is lower than $r_{7}$. The lowest error rate and highest error rate are $5.9723 \%$ and $36.1099 \%$, respectively, in $r_{7}$ of "Image 07 " and $r_{1}$ of "Image 09." Generally, the average error rate monotonously increases from high bit-plane to low bitplane. The average error rate for an entire image is $19.2673 \%$.

The compression ratio is greatly dependent on the error rate and the distribution of error bits. A residual bit-plane 


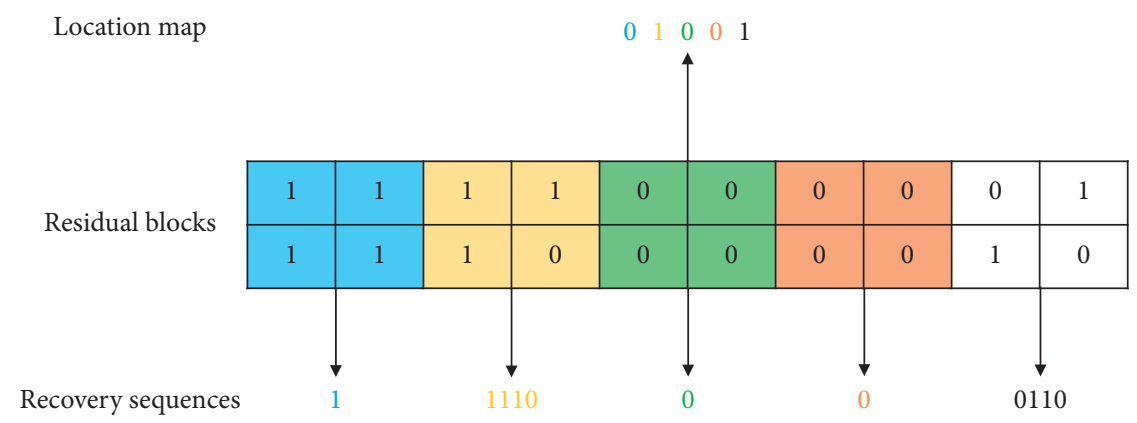

FIGURE 5: An example of the proposed adaptive residual bit-plane compression.

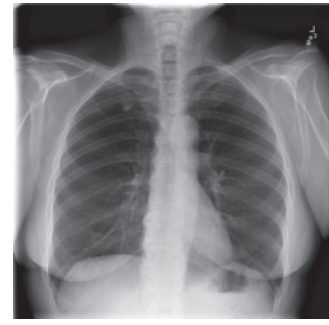

(a)

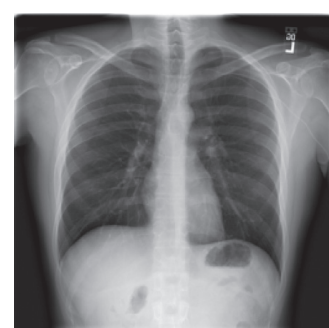

(f)

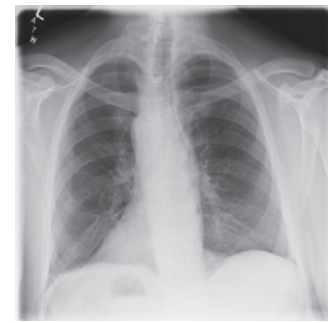

(b)

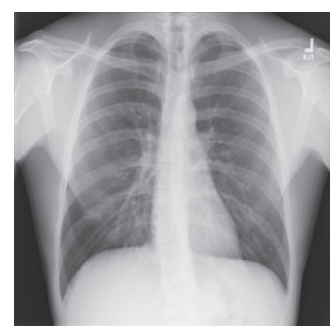

(g)

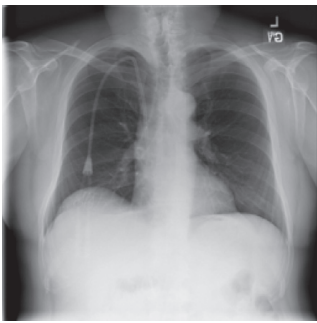

(c)

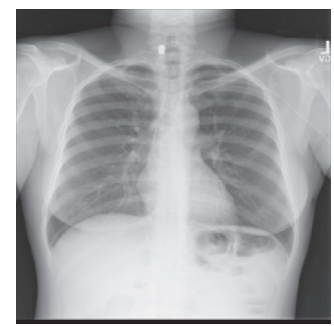

(h)

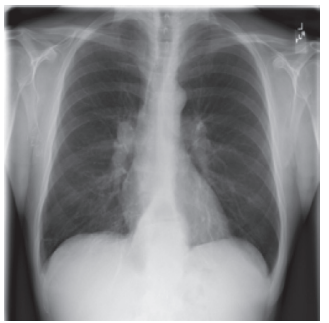

(d)

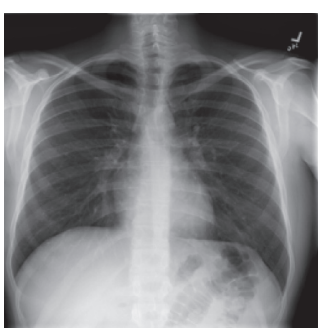

(i)

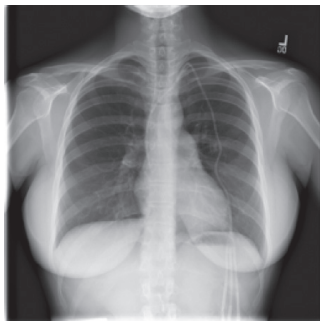

(e)

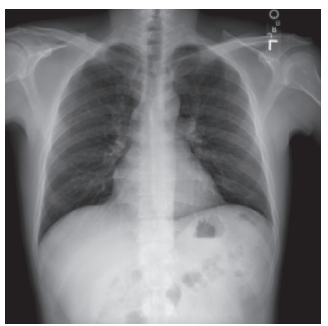

(j)

Figure 6: Ten test images: (a) Image 01, (b) Image 02, (c) Image 03, (d) Image 04, (e) Image 05, (f) Image 06, (g) Image 07, (h) Image 08, (i) Image 09, and (j) Image 10.

with a few concentrated error bits can be compressed efficiently. The compression ratio of the residual bit-planes is plotted in Figure 8. Since the number of error bits increases from $r_{7}$ to $r_{1}$, the compression ratio decreases as the bit layer decreases. Even in the exception cases of "Image 04" and "Image 06," where the error bits of $r_{7}$ are more than $r_{6}$, the compression ratio of $r_{7}$ is also greater due to the concentrated distribution of error bits.

To investigate the effect of block size setting, four different sizes are applied to compress the residual. The compression ratio for different block sizes and bit layers is listed in Table 2. The compression ratio of size 4 is significantly greater than the other sizes for all layers of bitplanes.

Additionally, our proposed residual bit-plane compression technique is also compared with the arithmetic coding technique. As shown in Figure 9, the average compression ratio of our proposed scheme is significantly better in the bit-planes of $r_{7}$ and $r_{6}$, while the performance of arithmetic coding is slightly better in the bit-planes of $r_{4}$ and $r_{3}$. That is because, in the rear residual bit-planes such as $r_{4}$ to $r_{1}$, the errors have no continuous distribution to make our compression technique not efficient. For compression of an entire image, the proposed scheme is significantly better than the arithmetic coding. To further verify the applicability in the medical image domain, the compression ratio for all test images is listed in Table 3. In Table $3, \mathscr{R}$ and $\mathscr{B}$ denote that the sources of compression are residuals and original bit-planes, respectively. Additionally, since the original first bit-plane should be transmitted at the beginning, in two types of bit-plane $\mathscr{R}$ and $\mathscr{B}$, the original first bit-plane is transmitted and compressed by different compression methods. As shown in Table 3, the compression ratio of residual is greater than that of the original version. It implies the devised GAN predictor bank is helpful. In addition, the proposed bitplane compression technique outperforms the arithmetic coding in the scheme of bit-planewise compression. As 


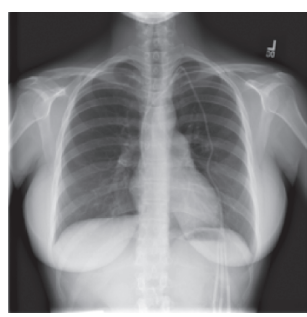

Original image

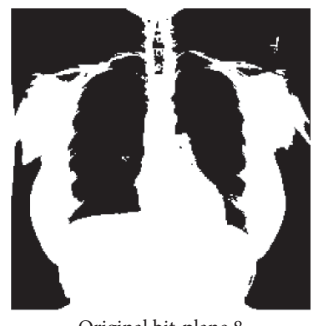

Original bit-plane 8

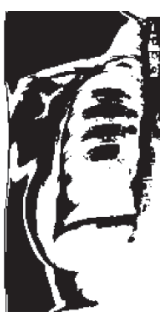

Original bit-plane

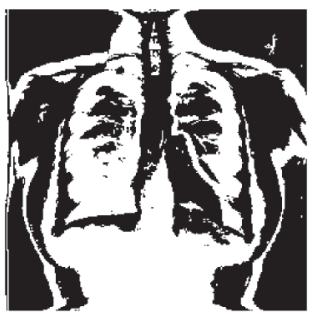

Predicted bit-plane 7

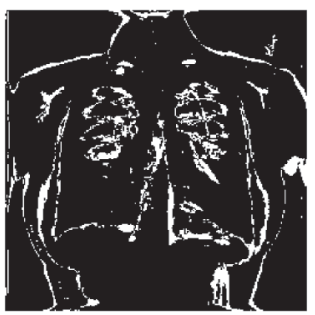

$9.5856 \%$

residual bit-plane 7

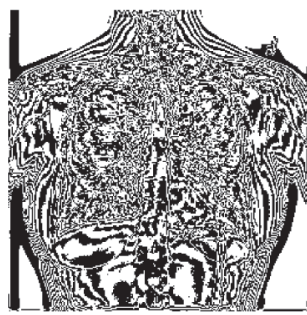

Original bit-plane 4

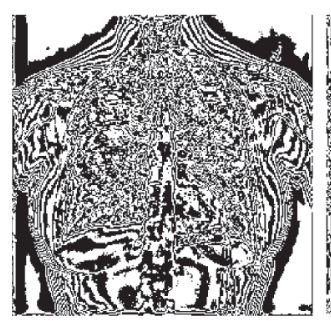

Predicted bit-plane 4

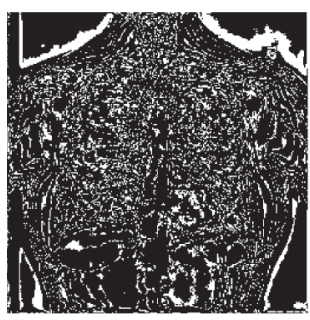

$19.2261 \%$

residual bit-plane 4

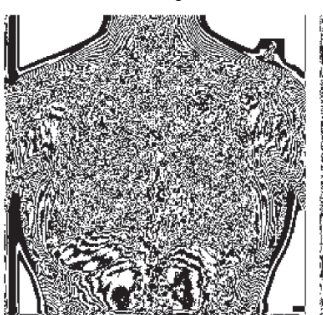

Original bit-plane 3

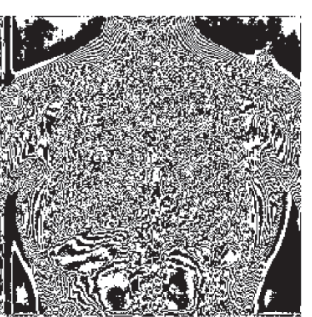

Predicted bit-plane 3

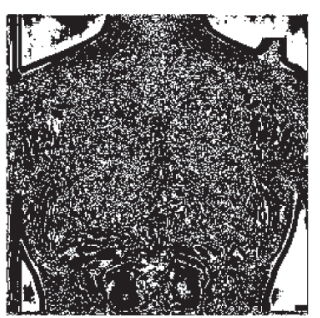

$26.5549 \%$

residual bit-plane 3

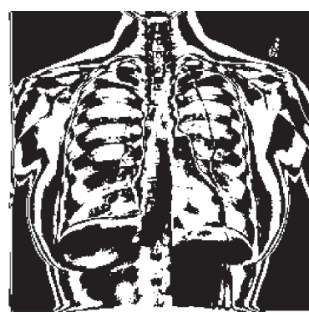

Original bit-plane 6

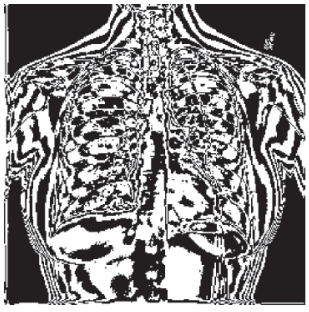

Original bit-plane 5

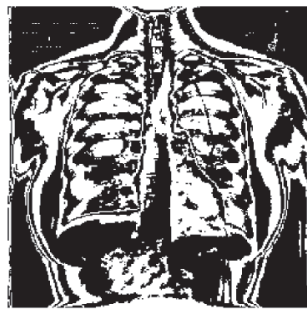

Predicted bit-plane 6

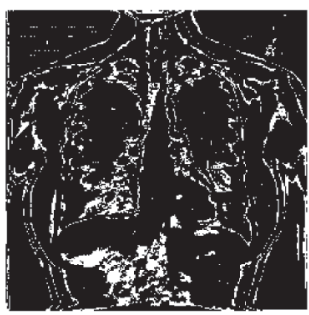

$10.9573 \%$

residual bit-plane 6

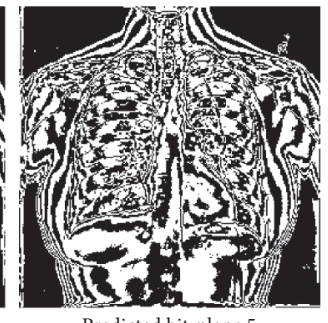

Predicted bit-plane 5

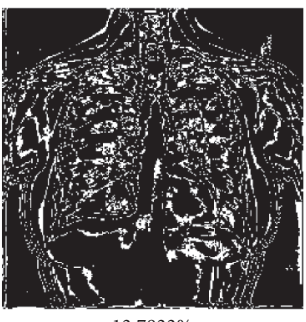

$13.7833 \%$

residual bit-plane

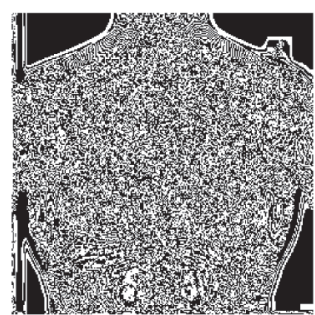

Original bit-plane 2

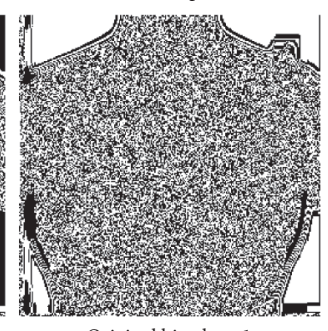

Original bit-plane 1

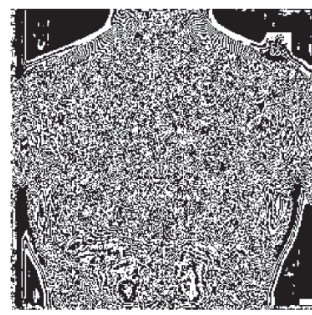

Predicted bit-plane 2

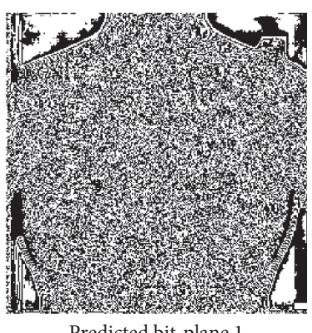

Predicted bit-plane 1

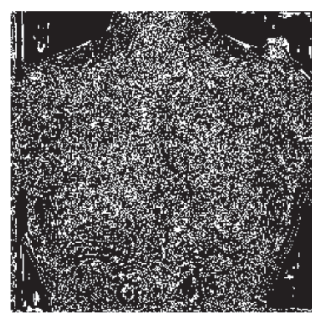

$24.6841 \%$

residual bit-plane 2

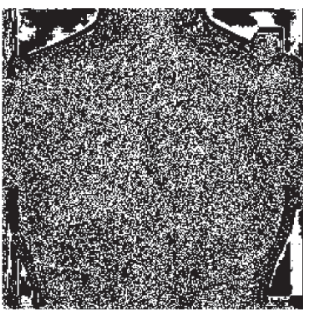

$33.3908 \%$

residual bit-plane

FIgURE 7: The original bit-planes (first row), the prediction bit-planes (second row), and the corresponding residual bit-planes with error rates (third row) of the test image "Image 05" (top-left). 
TABLE 1: Error rates ER of residual bit-planes 7-1 in test images.

\begin{tabular}{|c|c|c|c|c|c|c|c|c|}
\hline Images & $r_{7}$ & $r_{6}$ & $r_{5}$ & $r_{4}$ & $r_{3}$ & $r_{2}$ & $r_{1}$ & Average \\
\hline Image 01 & 10.4553 & 12.5137 & 14.1861 & 17.9123 & 22.0245 & 26.9897 & 34.8251 & 19.8438 \\
\hline Image 02 & 11.8851 & 14.6881 & 15.9241 & 18.6523 & 21.9116 & 25.9674 & 31.4346 & 20.0662 \\
\hline Image 03 & 8.4518 & 10.7819 & 12.5763 & 18.4158 & 21.4813 & 24.8047 & 31.7764 & 18.3269 \\
\hline Image 04 & 13.2202 & 11.4502 & 14.5264 & 18.1915 & 21.5698 & 26.7654 & 34.0698 & 19.9705 \\
\hline Image 05 & 9.5856 & 10.9573 & 13.7833 & 19.2261 & 26.5549 & 24.6841 & 33.3908 & 19.7403 \\
\hline Image 06 & 10.2814 & 9.4879 & 13.6139 & 22.3846 & 22.3145 & 26.4420 & 33.3206 & 19.6921 \\
\hline Image 07 & 5.9723 & 9.6817 & 14.3890 & 16.8106 & 20.3964 & 25.3586 & 31.3263 & 17.7050 \\
\hline Image 08 & 11.3373 & 11.6348 & 12.8296 & 16.2766 & 19.9966 & 24.0417 & 31.6544 & 18.2530 \\
\hline Image 09 & 16.1194 & 12.0071 & 14.6622 & 17.5919 & 21.8216 & 27.3041 & 36.1099 & 20.8023 \\
\hline Image 10 & 10.5713 & 12.0087 & 13.3682 & 16.1804 & 20.0516 & 24.5636 & 31.1661 & 18.2728 \\
\hline Average & 10.7880 & 11.5211 & 13.9859 & 18.1642 & 21.8123 & 25.6921 & 32.9074 & 19.2673 \\
\hline
\end{tabular}

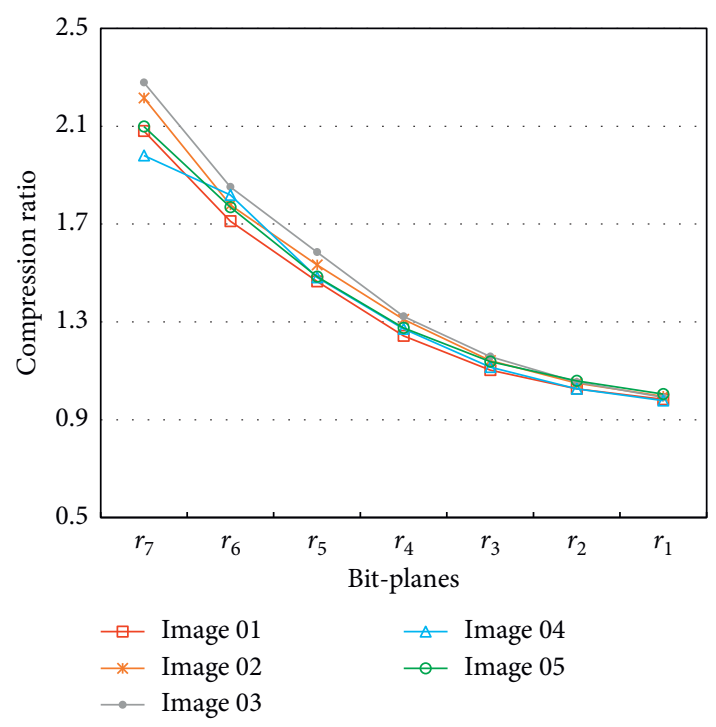

(a)

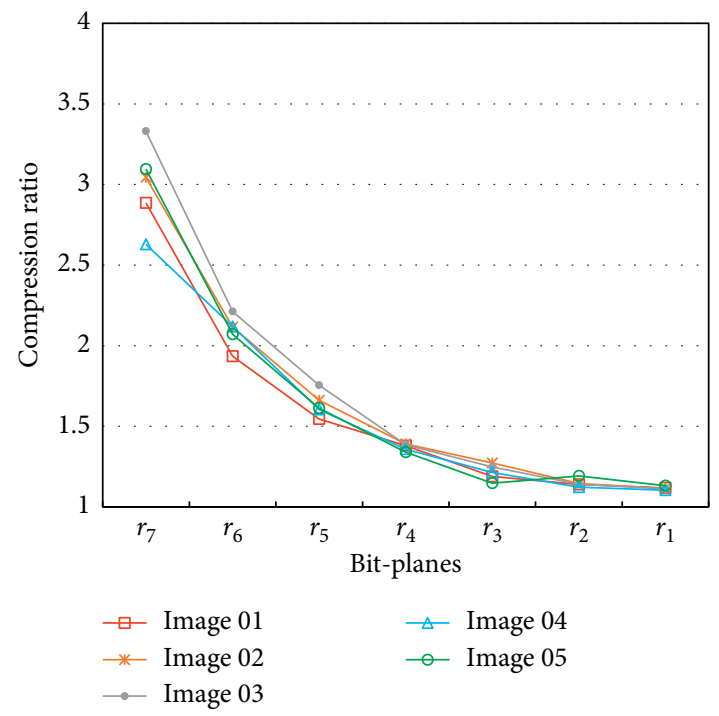

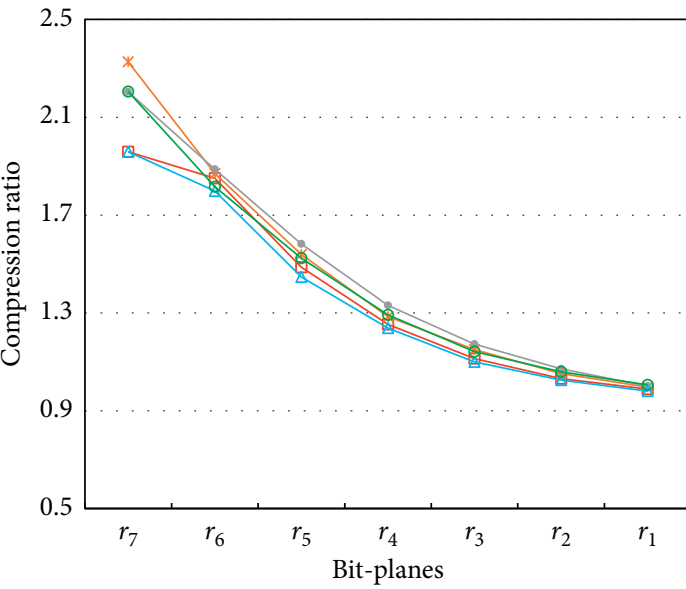

$$
\begin{aligned}
& \square \text { Image } 06 \quad \triangle \quad \text { Image } 09 \\
& \text { * Image } 07 \quad \text { - Image 10 }
\end{aligned}
$$$$
\rightarrow \text { Image } 08
$$

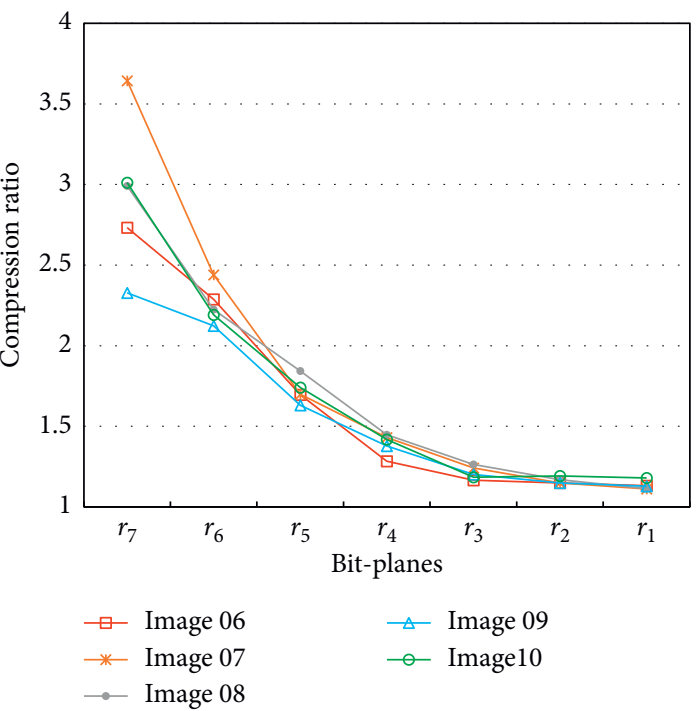

(b)

Figure 8: Continued. 

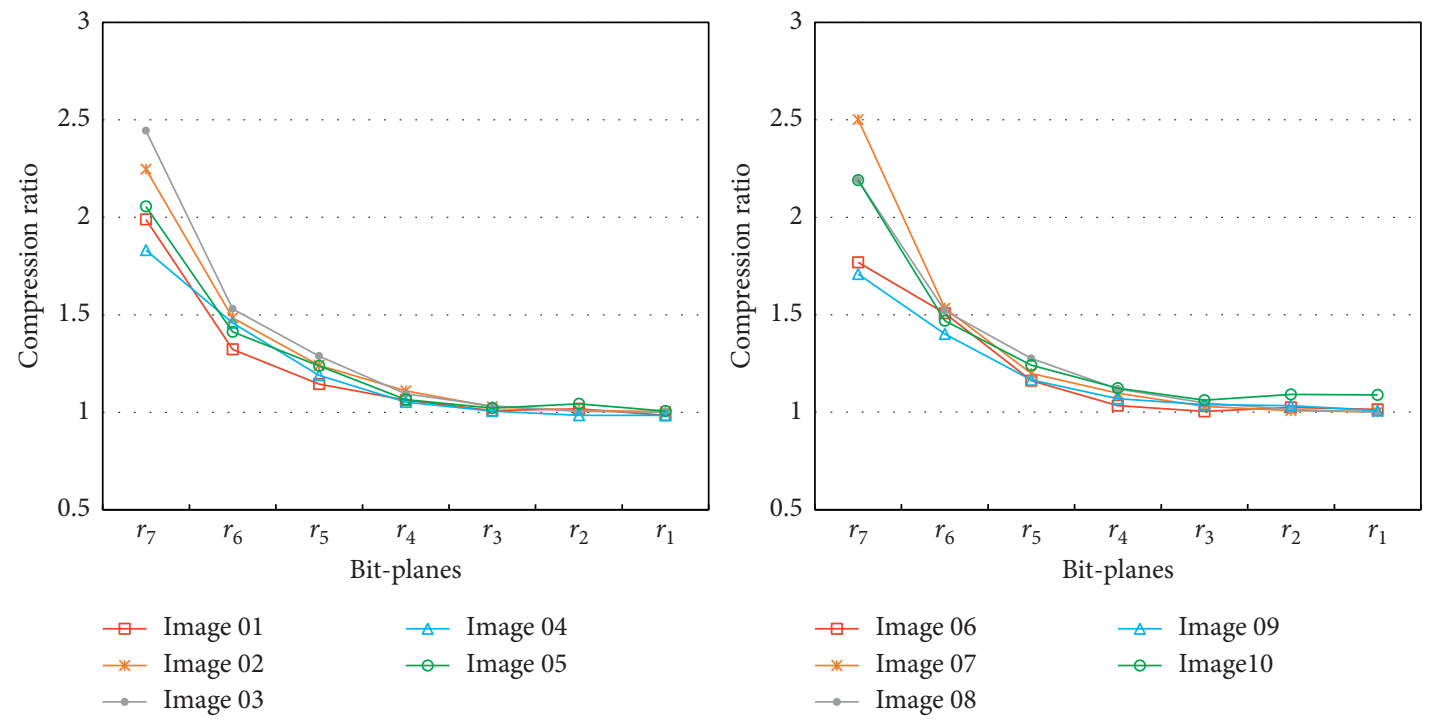

(c)
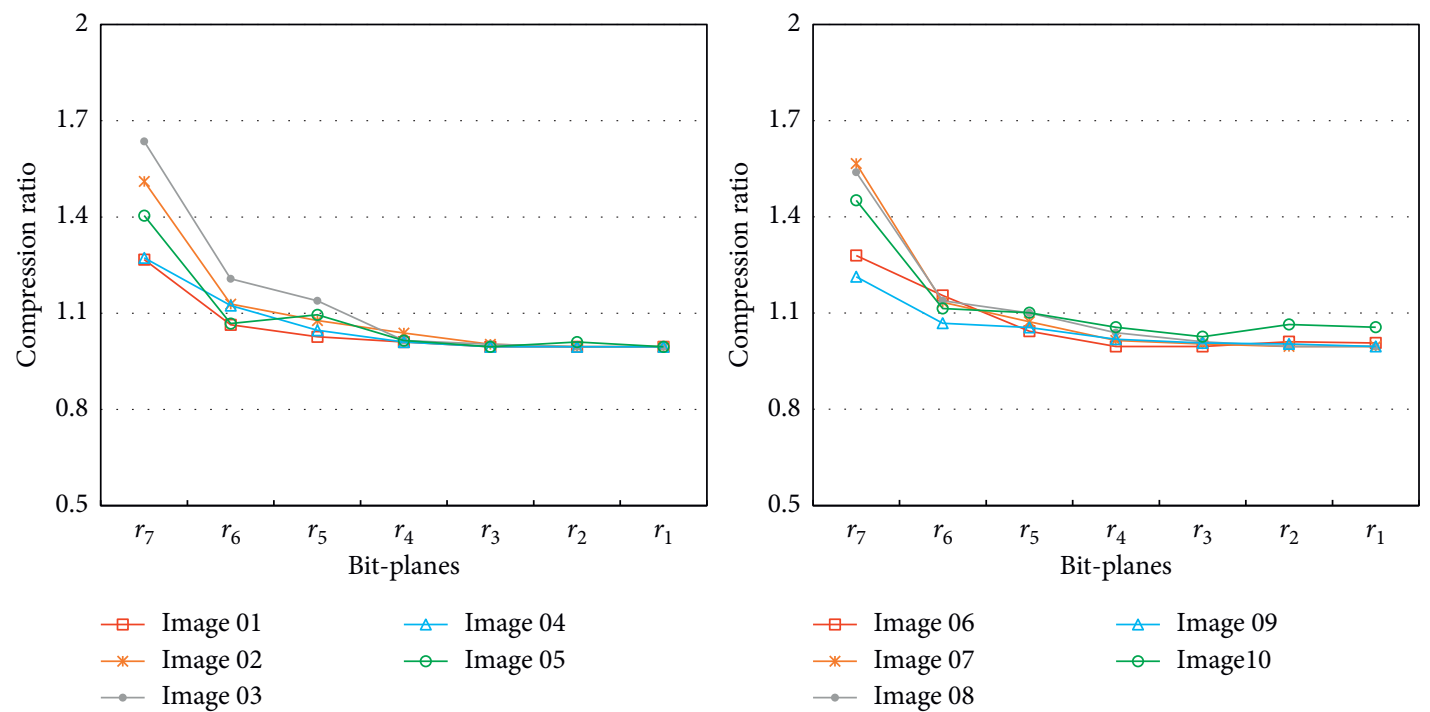

(d)

Figure 8: Compression ratio of each bit-plane in ten test images when the block sizes are (a) $m=2$, (b) $m=4$, (c) $m=8$, and (d) $m=16$.

TABle 2: Average bit-plane compression ratio (CR) for different block sizes.

\begin{tabular}{lcccccr}
\hline Block size & $r_{7}$ & $r_{6}$ & $r_{5}$ & $r_{4}$ & $r_{3}$ & $r_{2}$ \\
\hline 2 & 2.1295 & 1.8124 & 1.5112 & 1.2814 & 1.1327 & 1.0449 \\
4 & $\mathbf{2 . 9 0 9 5}$ & $\mathbf{2 . 2 0 5 9}$ & $\mathbf{1 . 7 0 5 3}$ & $\mathbf{1 . 3 7 3 1}$ & $\mathbf{1 . 2 6 1 4}$ & $\mathbf{1 . 2 1 3 7}$ \\
8 & 2.1585 & 1.5553 & 1.2788 & 1.1166 & 1.0435 & 1.0313 \\
16 & 1.4372 & 1.1383 & 1.0775 & 1.0266 & 1.0036 & 1.00722 \\
\hline
\end{tabular}

shown in the experimental results, the compression ratio is severely affected by the smoothness of residual bit-planes. In medical images, most of the textures are concentrated in the region of interest, which is particularly suitable for the proposed predictor bank to work efficiently. Additionally, the parameters of the pretrained predictor bank are crucial for the sender and receiver, so that the predictor bank needs to be protected to ensure applicability and security. 


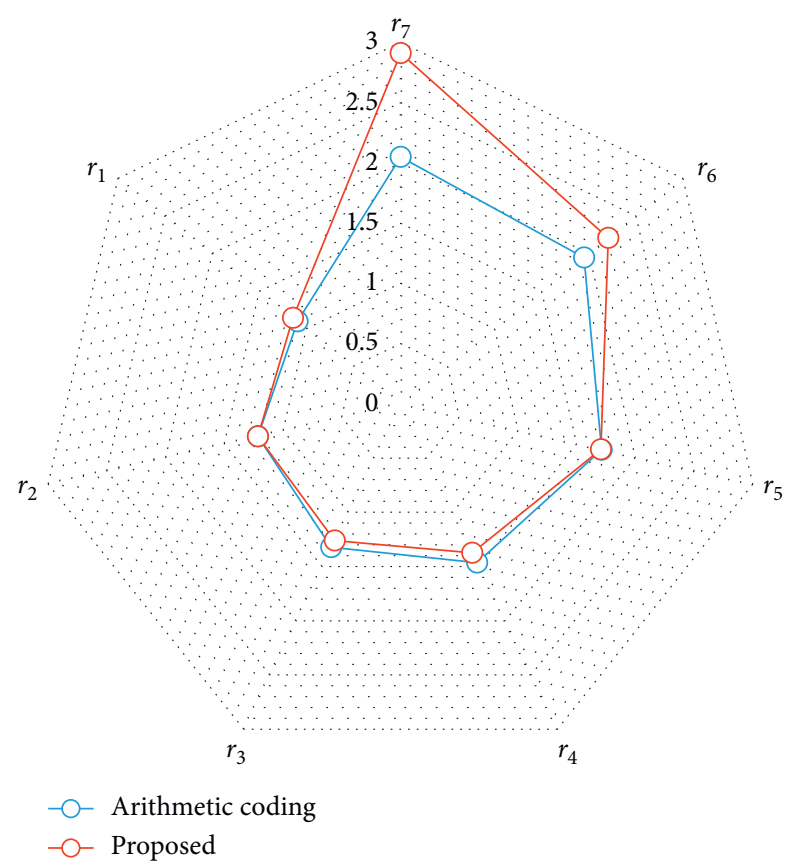

Figure 9: Average compression ratio comparison in each bit-plane.

TABLE 3: Comparison of compression ratio for ten test images.

\begin{tabular}{|c|c|c|c|c|c|c|c|c|c|c|}
\hline & \multicolumn{2}{|c|}{ Image 01} & \multicolumn{2}{|c|}{ Image 02} & \multicolumn{2}{|c|}{ Image 03} & \multicolumn{2}{|c|}{ Image 04} & \multicolumn{2}{|c|}{ Image 05} \\
\hline & $\mathscr{R}$ & $\mathscr{B}$ & $\mathscr{R}$ & $\mathscr{B}$ & $\mathscr{R}$ & $\mathscr{B}$ & $\mathscr{R}$ & $\mathscr{B}$ & $\mathscr{R}$ & $\mathscr{B}$ \\
\hline Proposed & 1.3923 & 1.2352 & 1.4347 & 1.3180 & 1.4566 & 1.3087 & 1.3970 & 1.2453 & 1.4272 & 1.2650 \\
\hline \multirow[t]{3}{*}{ Arithmetic coding } & 1.3739 & 1.0062 & 1.3475 & 1.0098 & 1.4255 & 1.0020 & 1.3629 & 1.0022 & 1.3783 & 1.0101 \\
\hline & \multicolumn{2}{|c|}{ Image 06} & \multicolumn{2}{|c|}{ Image 07} & \multicolumn{2}{|c|}{ Image 08} & \multicolumn{2}{|c|}{ Image 09} & \multicolumn{2}{|c|}{ Image 10} \\
\hline & $\mathscr{R}$ & $\mathscr{B}$ & $\mathscr{R}$ & $\mathscr{B}$ & $\mathscr{R}$ & $\mathscr{B}$ & $\mathscr{R}$ & $\mathscr{B}$ & $\mathscr{R}$ & $\mathscr{B}$ \\
\hline Proposed & 1.4003 & 1.2409 & 1.4609 & 1.2722 & 1.4615 & 1.2953 & 1.3917 & 1.2376 & 1.4601 & 1.2835 \\
\hline Arithmetic coding & 1.3827 & 1.0052 & 1.4585 & 1.0051 & 1.4166 & 1.0006 & 1.3349 & 1.0055 & 1.4158 & 1.0116 \\
\hline
\end{tabular}

\section{Conclusions}

In this paper, we introduce a GAN predictor bank and a bitplane compression technique into the progressive transmission of medical images. Experimental results validate the proposed predictor bank can effectively predict bit-planes under the progressive scheme. Furthermore, the proposed adaptive bit-plane compression is more efficient than the arithmetic coding for the current application. This paper intends to introduce deep learning techniques into the classical progressive image transmission and we hope this paper can give some illuminations for future research. In our future works, we will further introduce and improve more convolutional neural networks into the compression and transmission of medical images.

\section{Data Availability}

The data used to support the findings of the study are available from the corresponding author upon request.

\section{Conflicts of Interest}

The authors declare that they have no conflicts of interest.

\section{Acknowledgments}

This work was partially supported by the JSPS KAKENHI (Grant nos. JP16H06302 and JP18H04120) and JST CREST (Grant nos JPMJCR18A6 and JPMJCR20D3), Japan.

\section{References}

[1] H. U. Lemke, "Communication networks for medical image transmission," Strahlentherapie und Onkologie: Organ der Deutschen Röntgengesellschaft, vol. 169, no. 9, pp. 512-520, 1993.

[2] R. Maani, S. Camorlinga, N. Arnason, and R. Eskicioglu, "A practical fast method for medical imaging transmission based on the DICOM protocol," Medical Imaging 2010: Advanced PACS-Based Imaging Informatics and Therapeutic Applications, vol. 7628, Article ID 76280M, 2010.

[3] R. Maani, S. Camorlinga, and N. Arnason, "A parallel method to improve medical image transmission," Journal of Digital Imaging, vol. 25, no. 1, pp. 101-109, 2012.

[4] K.-H. Tzou, "Progressive image transmission: a review and comparison of techniques," Optical Engineering, vol. 26, no. 7, Article ID 267581, 1987.

[5] C.-C. Chang, F.-C. Shiue, and T.-S. Chen, "A new scheme of progressive image transmission based on bit-plane method," 
in Proceedings of the Fifth Asia-Pacific Conference on and Fourth Optoelectronics and Communications Conference on Communications, pp. 892-895, Singapore, October 1999.

[6] T.-S. Chen, H.-C. Wu, H.-F. Tsai, M. Hsieh, and S.-F. Chiou, "Progressive transmission of two-dimensional gel electrophoresis image based on context features and bit-plane method," in Proceedings of the IEEE International Conference on Networking, Sensing and Control, pp. 1241-1246, Taipei, Taiwan, March 2004.

[7] L. Wang and M. Goldberg, "Progressive image transmission using vector quantization on images in pyramid form," IEEE Transactions on Communications, vol. 37, no. 12, pp. 13391349, 1989.

[8] C.-C. Chang, H.-C. Hsia, and T.-S. Chen, "A progressive image transmission scheme based on block truncation coding," in The Human Society and the Internet Internet-Related Socio-Economic Issuespp. 383-397, Berlin, Heidelberg, 2001.

[9] K.-L. Chung and S.-Y. Tseng, "New progressive image transmission based on quadtree and shading approach with resolution control," Pattern Recognition Letters, vol. 22, no. 14, pp. 1545-1555, 2001.

[10] C.-C. Chang, G.-X. Xiao, and T.-S. Chen, "A simple prediction method for progressive image transmission," in Distributed Multimedia Databases: Techniques and Applications, 2002, The Idea Group Publishing, Hershey, PA, USA, ht tp://www.igi-global.com/chapter/distributed-multimedia-dat abases/8626.

[11] H. Kim, R. Annavajjala, P. Cosman, and L. Milstein, "Sourcechannel rate optimization for progressive image transmission over block fading relay channels," IEEE Transactions on Communications, vol. 58, no. 6, pp. 1631-1642, 2010.

[12] B. C. Dhara and B. Chanda, "A fast progressive image transmission scheme using block truncation coding by pattern fitting," Journal of Visual Communication and Image Representation, vol. 23, no. 2, pp. 313-322, 2012.

[13] L. Wang and M. Goldberg, "Progressive image transmission by transform coefficient residual error quantization," IEEE Transactions on Communications, vol. 36, no. 1, pp. 75-87, 1988.

[14] W. J. Hwang and H. Derin, "Multiresolution multiresource progressive image transmission," IEEE Transactions on Image Processing, vol. 4, no. 8, pp. 1128-1140, 1995.

[15] Y. Lu, J. Zheng, Y. Jiang, M. Yang, B. Fu, and W. Hou, "Progressive image transmission for medical applications based on wavelet transform with a non-uniform scalar quantization scheme," in Proceedings of the 2006 International Conference of the IEEE Engineering in Medicine and Biology Society, pp. 4795-4798, New York, NY, USA, August 2006.

[16] C.-C. Chang and T.-C. Lu, "A wavelet-based progressive digital image transmission scheme," in Proceedings of the First International Conference on Innovative Computing, Information and Control-Volume I (ICICIC'06), pp. 681-684, Beijing, China, September 2006.

[17] C.-P. Huang and C.-C. Li, "Secure and progressive image transmission through shadows generated by multiwavelet transform," International Journal of Wavelets, Multiresolution and Information Processing, vol. 6, no. 6, pp. 907-931, 2008.

[18] R. S. Dilmaghani, A. Ahmadian, M. Ghavami, and A. H. Aghvami, "Progressive medical image transmission and compression," IEEE Signal Processing Letters, vol. 11, no. 10, pp. 806-809, 2004.

[19] K.-L. Hung, C.-C. Chang, and I.-C. Lin, "Lossless compression-based progressive image transmission scheme," The Imaging Science Journal, vol. 52, no. 4, pp. 212-224, 2004.
[20] Y. LeCun, Y. Bengio, and G. Hinton, “Deep learning," Nature, vol. 521, no. 7553, pp. 436-444, 2015.

[21] R. Neelapu, G. Lavanya Devi, and K. Srinivasa Rao, "Deep learning based conventional neural network architecture for medical image classification," Traitement du signal, vol. 35, no. 2, pp. 169-182, 2018.

[22] L. Faes, S. K. Wagner, D. J. Fu et al., "Automated deep learning design for medical image classification by health-care professionals with no coding experience: a feasibility study," The Lancet Digital Health, vol. 1, no. 5, pp. e232-e242, 2019.

[23] J. Wagner, Y. Xie, Q. Wu, and Y. Xia, "Medical image classification using synergic deep learning," Medical Image Analysis, vol. 54, pp. 10-19, 2019.

[24] H. Chen, D. Ni, J. Qin et al., "Standard plane localization in fetal ultrasound via domain transferred deep neural networks," IEEE Journal of Biomedical and Health Informatics, vol. 19, no. 5, pp. 1627-1636, 2015.

[25] Y. Ni, M. Landis, D. T. Laidley, A. Kornecki, A. Lum, and S. Li, "Multi-modal vertebrae recognition using transformed deep convolution network," Computerized Medical Imaging and Graphics, vol. 51, pp. 11-19, 2016.

[26] A. Kumar, "Plane identification in fetal ultrasound images using saliency maps and convolutional neural networks," in Proceedings of the 2016 IEEE 13th International Symposium on Biomedical Imaging (ISBI), pp. 791-794, Prague, Czech Republic, April 2016.

[27] A. Teramoto, H. Fujita, O. Yamamuro, and T. Tamaki, "Automated detection of pulmonary nodules in PET/CT images: ensemble false-positive reduction using a convolutional neural network technique," Medical Physics, vol. 43, no. 6, pp. 2821-2827, 2016.

[28] M. J. J. P. Van Grinsven, B. van Ginneken, C. B. Hoyng, T. Theelen, and C. I. Sánchez, "Fast convolutional neural network training using selective data sampling: application to hemorrhage detection in color fundus images," IEEE Transactions on Medical Imaging, vol. 35, no. 5, pp. 1273-1284, 2016.

[29] J. M. Wolterink, T. Leiner, B. D. de Vos, R. W. van Hamersvelt, M. A. Viergever, and I. Išgum, "Automatic coronary artery calcium scoring in cardiac CT angiography using paired convolutional neural networks," Medical Image Analysis, vol. 34, pp. 123-136, 2016.

[30] P. F. Christ, "Automatic liver and lesion segmentation in CT using cascaded fully convolutional neural networks and 3D conditional random fields," in Proceedings of the International Conference on Medical Image Computing and ComputerAssisted Intervention, pp. 415-423, Athens, Greece, October 2016.

[31] M. Gao, "Segmentation label propagation using deep convolutional neural networks and dense conditional random field," in Proceedings of the 2016 IEEE 13th International Symposium on Biomedical Imaging (ISBI), pp. 1265-1268, Prague, Czech Republic, April 2016.

[32] K. Kamnitsas, C. Ledig, V. F. J. Newcombe et al., "Efficient multi-scale 3D CNN with fully connected CRF for accurate brain lesion segmentation," Medical Image Analysis, vol. 36, pp. 61-78, 2017.

[33] Y. Ledig, I. Kogan, E. Gelbart, O. Geva, and H. Greenspan, "Visualizing and enhancing a deep learning framework using patients age and gender for chest x-ray image retrieval," in Proceedings of the SPIE on Medical Imaging, p. 978510, San Diego, CA, USA, 2016.

[34] A. Janowczyk, A. Basavanhally, and A. Madabhushi, "Stain normalization using sparse autoencoders (StaNoSA): 
application to digital pathology," Computerized Medical Imaging and Graphics, vol. 57, pp. 50-61, 2017.

[35] P. Kisilev, E. Sason, E. Barkan, and S. Hashoul, "Medical image description using multi-task-loss CNN," in Deep Learning and Data Labeling for Medical Applications, Springer, Cham, Switzerland, 2016.

[36] I. J. Goodfellow, "Generative adversarial networks," ArXiv14062661 Cs Stat, 2014, http://arxiv.org/abs/1406.2661.

[37] P. Isola, J.-Y. Zhu, T. Zhou, and A. A. Efros, "Image-to-image translation with conditional adversarial networks," in Proceedings of the IEEE Conference on Computer Vision and Pattern Recognition, pp. 1125-1134, Honolulu, HI, USA, July 2017.

[38] C.-C. Chang, "Adversarial learning for invertible steganography," IEEE Access, vol. 8, pp. 198425-198435, 2020.

[39] M. Sato, K. Hotta, A. Imanishi, M. Matsuda, and K. Terai, "Segmentation of cell membrane and nucleus by improving Pix2pix," Biosignals, pp. 216-220, 2018.

[40] M. Mori, T. Fujioka, L. Katsuta et al., "Feasibility of new fat suppression for breast MRI using pix2pix," Japanese Journal of Radiology, vol. 38, no. 11, pp. 1075-1081, 2020. 